Das Bildungswesen steht somit weitgehend im Dienste der außenorientierten modernen Wirtschaftssektoren. Es dient der Rekrutierung und Reproduktion der Elite. Es fördert und verstärkt die Marginalisierung und die Landflucht und hat keinen Bezug zu den Lebensbedingungen der Masse der Bevölkerung. Eine Lösung dieser Probleme hält die Autorin allerdings - wohl zu Recht - auch nicht allein durch isolierte Bildungsreformen - wie etwa durch die Ruralisierung der Curricula der Primärschulen, wie jüngst geschehen - für möglich. Sie sind ihrer Meinung nach bestenfalls geeignet, ,,die Masse der Bevölkerung von dem existenzgefährdenden Konsumverzicht, herkömmlicher moderner Bildung' zu befreien, der für sie in dem gegebenen System nicht Produktivität, sondern Unproduktivität zur Folge hat!" (S. 338). Erst eine Bildungsreform im Rahmen einer auch in anderen Bereichen veränderten gesamtgesellschaftlichen Entwicklungsstrategie, etwa durch eine tatsächliche Konzentration auf die Landwirtschaft und die rurale Entwicklung, würde in der Lage sein, die Entwicklungsdefizite und Dysfunktionalitäten zu überwinden.

Eine nicht nur für Bildungsökonomen und Kamerun-Spezialisten lesenswerte Arbeit.

Rolf Hanisch

Riaz Hassan (Herausgeber)

Singapore: Society in Transition

Kuala Lumpur, London, New York, Melbourne 1976, 371 Seiten.

Für viele Betrachter erscheint Singapore als ein Staat, der sich wegen seines Entwicklungsstandes vor allem im wirtschaftlichen Bereich, seiner politischen und sozialen Stabilität, der effizienten Verwaltung und Infrastruktur grundlegend von den anderen Südost-asiatischen Staaten unterscheidet und zwar im positiven Sinne. Das vorliegende Werk verhilft zu einer differenzierteren Betrachtung. 16 Autoren verschiedener Fachbereiche untersuchen die politischen, sozialen, ökonomischen und kulturellen Verhältnisse und deren Entwicklung in Singapore. 28 Seiten sind dem Rechtssystem gewidmet. Volumenmäßig liegt der Schwerpunkt des Compendiums (220 Seiten) bei Themen des sozialen und kulturellen Bereichs. Alle Aufsätze zeichnen sich durch eine knappe, aber gründliche, informative und interessante Darstellung aus. Die Analysen lassen die weniger sichtbaren, aber für die Zukunft möglicherweise brisanten Schwächen des ,,Modellstaates“ erkennen. Die Autoren rufen ins Bewußtsein, daß die Stabilität des autoritären Einparteienstaates, die Ausrichtung des Erziehungssystems auf die wirtschaftliche Zielsetzung und die immer noch auf der Rezeption englischen Rechts beruhende Rechtsordnung die Entwicklung einer wirklichen ,,Identität“ erschweren.

Die Autoren weisen auf Schwächen hin, aber sie prangern nicht an, vielmehr untersuchen sie die zweifellos überwiegend positive Entwicklung Singapores und stellen die sich für die $\mathrm{Zu}$ kunft ergebenden Aufgaben fest. Das Werk kann in jeder Hinsicht empfohlen werden.

K. Kähler

Von Ernesto De La Torre Villar/Jorge Mario Garcia Laguardia

Desarollo histórico del constitucionalismo hispanoamericano. Serie B. Estudios comparativos. Derecho latinamericano Núm. 11. Instituto de Investigaciones Jurídicas de la Universidad Autónoma de México, México 20. D. F. 1976, 314 S.

Die gerade in Lateinamerika immer häufiger zu beobachtende Skepsis - wenn nicht gar betont kritische Einstellung - gegenüber der etablierten auswärtigen Entwicklungsländerforschung unterstreicht, wie notwendig es ist, deren wachsenden l'art-pour-l'art-Trend durch 
verstärkte Berücksichtigung einheimischer Forschungsergebnisse gegenzusteuern. Die vorliegende Gemeinschaftsarbeit zweier renommierter lateinamerikanischer Staatsrechtler ( $T$. V. ist Mexikaner, G. L. Guatemalteke) vermag hierfür wertvolle Impulse zu geben. Sie bietet allerdings, trotz des anspruchsvoll weit gefaßten Titels, keineswegs eine komplette Verfassungsgeschichte Spanisch-Amerikas, sondern versteht sich als vergleichende Studie lediglich zur spät- und nachkolonialen Verfassungsentwicklung eines zudem begrenzten Raumes, nämlich Mexikos und der zentralamerikanischen Isthmus-Staaten. Nur unter bestimmten Aspekten wie etwa ,Liberalismus“ und ,,sozialer Konstitutionalismus“ sind auch Cuba (S. 214-220, 268-276) und Puerto Rico (S. 221-224) einbezogen. Innerhalb dieser Limitierung spannt sich der Bogen von den mexikanischen Anfängen der Unabhängigkeitsbewegung 1808 bis zu den Vorarbeiten für die cubanische Verfassung von 1976. Den möglicherweise als Mangel empfundenen Verzicht auf verfassungsrechtliche Details kompensieren die Autoren vollauf durch die verstärkte Einbeziehung politischer und sozio-ökonomischer Faktoren. Deren Beurteilung mag mitunter einige Wünsche offen lassen. Insgesamt jedoch erweist sich die gut lesbare Darstellung einschließlich ihrer über 400 Titel umfassenden Auswahlbibliographie - zusätzlich zum opulent mit weiteren Hinweisen bestückten Fußnoten-Apparat als wahre Fundgrube für jeden, dem an Originalquellen aus Lateinamerika eher gelegen ist als in der steigenden Flut von Sekundärliteratur über diesen Halbkontinent.

\section{JACQUeS VANDERLINDEN}

Karl Hernekamp

\section{L'Ethiopie et ses Populations}

Editions Complexe 1977, Brüssel 1977, 254 S.

Das Buch kommt gerade zu einem Zeitpunkt heraus, in dem sich die Weltöffentlichkeit mit dem Horn von Afrika intensiv beschäftigt. Der Autor, der auch an der ehemaligen Haile-Selassie-Universität unterrichtet hatte, ist mit dem Gegenstand gut vertraut, und auch mit Veröffentlichungen zum äthiopischen Recht bekannt geworden. Das vorliegende Werk befaßt sich aber mehr mit der Geschichte und der Ethnographie des äthiopischen Raumes. Nach der Einleitung behandelt der Verfasser auf ca. 45 Seiten die äthiopische Geschichte bis hin in die Revolution, widmet sich dann den äthiopischen Städten einschließlich der sozialen Verhältnisse der Erziehung, der Gewerkschaften, der Planung, der Investitionen und der Verwaltung. In diesem Rahmen wird auch das moderne Recht behandelt. Allerdings wird diesem Gegenstand kaum mehr als 1 1/2 Seiten gewidmet. Dem schließen sich Ausführungen über die Ethnien an, und zwar: Die Amharas (S. 90), die Gourages (S. 115), die Bevölkerung von Tigre (S. 123) - womit die semitischen Teile erfaßt sind-, die Oromo, die Sidamo, die Somali (S. 127, 142 und 153 ff.), womit die kuschitischen Bevölkerungsteile abgehandelt werden. Dann folgen Randstämme wie die Affar (S. 163), die Falacha und die Bevölkerung von Eritrea (S. 189).

Nach dieser Ubersicht kommt ein Ausblick in die Zukunft (S. 209); und zwar mit wirtschaftlichem, gesellschaftlichem und politischem Aspekt. Das Verhältnis der Bauern, der landwirtschaftlichen Technik und der Planung der Ausfuhr und der Industrie werden genauso knapp dargestellt wie Regionalismus und Zentralismus der Kultur und Ethnienpluralismus, die Stellung der Kirche, die Entwicklung einer neuen Klasse. Das Buch, das reiches Bildmaterial enthält, schließt mit einer allgemeinen Bibliographie ab, die nach den Kapiteln geordnet ist. Ein Stichwortverzeichnis und ein Glossar erleichtern das Arbeiten mit diesem Werk, das auch durch Rand- oder Marginalüberschriften übersichtlich gestaltet ist, aber nicht in die Tiefe geht.

Heinrich Scholler 